\title{
Datenschutz in Europa stärken
}

\section{Konferenz der Datenschutzbeauftragten des Bundes und der Länder am 13. und 14. März 2013 in Bremerhaven}

Die Konferenz der Datenschutzbeauftragten des Bundes und der Länder ist ein freiwilliger Zusammenschluss der amtlichen Datenschutzbeauftragten und stellt die gemeinsame Arbeitsebene der beteiligten Institutionen dar.Sie fand erstmalig am 7.Dezember 1978 in Hessen statt und tagt seitdem zweimal jährlich unter dem wechselnden Vorsitz eines Datenschutzbeauftragten.

Im Jahr 2013 werden die 85. und die 86. Konferenz unter Vorsitz der Landesbeauftragten für Datenschutz und Informationsfreiheit Bremen (LfDI Bremen) in Bremerhaven (13.03.-14.03.) und in Bremen (01.10.-02.10.) ausgerichtet.

Die Konferenz der Datenschutzbeauftragten des Bundes und der Länder hat sich auf ihrer 85. Tagung in Bremerhaven für die Stärkung des Datenschutzes auf europäischer Ebene eingesetzt. Die EUKommission hatte im vergangenen Jahr den Entwurf einer Datenschutz-Grundverordnung vorgelegt. Die Datenschutzbeauftragten wenden sich gegen alle Änderungsvorschläge, deren Umsetzung das Grundrecht auf Datenschutz schwächen würde. Die Konferenz appelliert in ihrer Entschließung „Europa muss den Datenschutz stärken "1 an das Europäische Parlament, den Rat und die Kommission das europäische Datenschutzgrundrecht wirksam zu gewährleisten. Insbesondere tritt sie für folgende Forderungen ein:

- Jedes personenbeziehbare Datum muss geschützt werden

- Es darf keine grundrechtsfreien Räume geben

- Einwilligungen müssen ausdrücklich erteilt werden

- Datenverarbeiter dürfen ihre Ziele nicht eigenmächtig verändern

- Profilbildungen müssen beschränkt werden

- Stärkung der Eigenverantwortung der Datenverarbeiter durch betriebliche Datenschutzbeauftragte

- Datenverarbeiter dürfen sich ihre Aufsichtsbehörde nicht aussuchen können

- Völlige Unabhängigkeit der Aufsichtsbehörden auch gegenüber der Kommission

- Grundrechtsschutz braucht effektive Kontrollen

- Hoher Datenschutzstandard für ganz Europa

Auch bei den jetzt beginnenden Verhandlungen zwischen der EU und den USA über eine transatlantische Freihandelszone muss sichergestellt werden, dass das in der Europäischen Grundrechtecharta garantierte Recht auf Datenschutz und die daraus abgeleiteten Standards gewahrt bleiben ${ }^{2}$.

Es zeichnet sich ab, dass die seit langem angekündigte Selbstregulierung der Betreiber sozialer Netzwerke aufgrund des Widerstandes großer Anbieter nicht zustande kommt. Die Konferenz fordert daher den Gesetzgeber in ihrer Entschließung „Soziale Netzwerke brauchen Leitplanken “3 nachdrücklich auf, die noch bestehenden Gesetzeslücken schnell zu schließen. Die Datenschutzbeauftragten haben eine Orientierungshilfe erarbeitet, die sich sowohl an Betreiber sozialer Netzwerke als auch an Behör-

1 DuD in diesem Heft S. 383

2 Ebenda, S. 383

3 Ebenda, S. 384 den oder Unternehmen wendet, die ihre Aufgaben mit Hilfe dieser Netzwerke erfüllen wollen.

Auf den Umstand, dass das gegenwärtig genutzte Verfahren für die Pseudonymisierung von Krebsregisterdaten etwa 20 Jahre alt ist, hat die Konferenz der Datenschutzbeauftragten des Bundes und der Länder mit der Entschließung: „Pseudonymisierung von Krebsregisterdaten verbessern ${ }^{\text {"4 }}$ reagiert. Darin fordert sie die zuständigen Fachaufsichtsbehörden der Länder auf, Rahmenbedingungen für eine dem heutigen Stand der Technik angemessene Pseudonymisierung zu schaffen. Mit der ständig gewachsenen Rechenkapazität sind auch die technischen Möglichkeiten zur Umkehrung der Pseudonymisierung und somit die Risiken für den Schutz der Patientendaten größer geworden. Die Datenschutzkonferenz formuliert in ihrer Entschließung Anforderungen an ein datenschutzgerechtes Verfahren zur Pseudonymisierung.

Auch hat die Konferenz eine engere Zusammenarbeit mit der Koordinierungsstelle für IT-Standards (KoSIT) vereinbart. Die KoSIT koordiniert und erstellt im Auftrag des IT-Planungsrates des Bundes und der Länder IT-Standards für die öffentlichen Verwaltungen.

\footnotetext{
4 Ebenda, S. 384
}

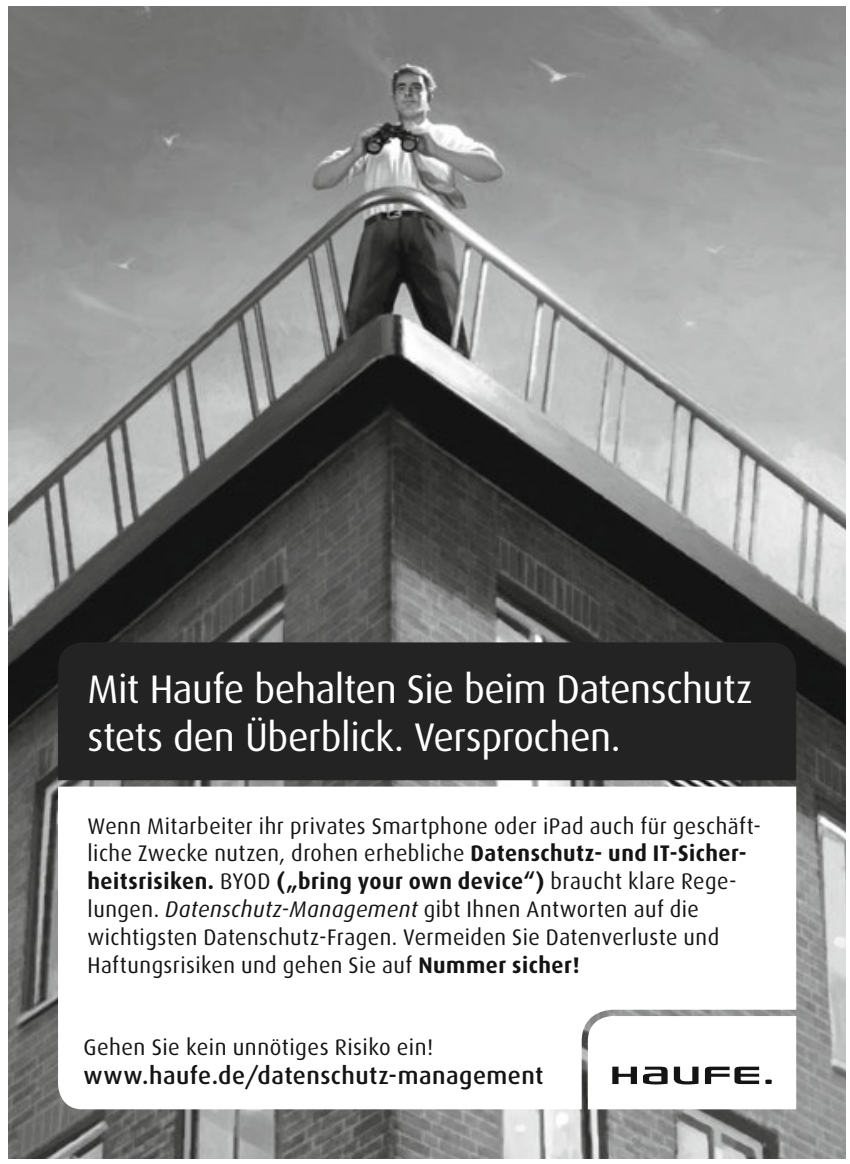

